Article

\title{
Wide Spectral Characteristics of Si Photonic Crystal Mach-Zehnder Modulator Fabricated by Complementary Metal-Oxide-Semiconductor Process
}

\author{
Yosuke Hinakura, Yosuke Terada, Takuya Tamura and Toshihiko Baba * \\ Department of Electrical and Computer Engineering, Yokohama National University, Yokohama 240-8501, Japan; \\ hinakura-yosuke-zm@ynu.jp (Y.H.); yterada@ynu.ac.jp (Y.T.); tamura-takuya-nt@ynu.jp (T.T.) \\ * Correspondence: baba@ynu.ac.jp; Tel.: +81-045-339-4258
}

Received: 29 February 2016; Accepted: 29 March 2016; Published: 2 April 2016

\begin{abstract}
Optical modulators for optical interconnects require a small size, small voltage, high speed and wide working spectrum. For this purpose, we developed Si slow-light Mach-Zehnder modulators via a $180 \mathrm{~nm}$ complementary metal-oxide-semiconductor process. We employed $200 \mu \mathrm{m}$ lattice-shifted photonic crystal waveguides with interleaved p-n junctions as phase shifters. The group index spectrum of slow light was almost flat at $n_{\mathrm{g}} \approx 20$ but exhibited $\pm 10 \%$ fluctuation over a wavelength bandwidth of $20 \mathrm{~nm}$. The cutoff frequency measured in this bandwidth ranged from 15 to $20 \mathrm{GHz}$; thus, clear open eyes were observed in the $25 \mathrm{Gbps}$ modulation. However, the fluctuation in $n_{\mathrm{g}}$ was reflected in the extinction ratio and bit-error rate. For a stable error-free operation, a $1 \mathrm{~dB}$ margin is necessary in the extinction ratio. In addition, we constructed a device with varied values of $n_{\mathrm{g}}$ and confirmed that the extinction ratio at this speed was enhanced by larger $n_{\mathrm{g}}$ up to 60 . However, this larger $n_{\mathrm{g}}$ reduced the cutoff frequency because of increased phase mismatch between slow light and radio frequency signals. Therefore, $n_{\mathrm{g}}$ available for $25 \mathrm{Gbps}$ modulation is limited to up to 40 for the current device design.
\end{abstract}

Keywords: silicon photonics; optical interconnect; photonic crystal; optical modulator; silicon modulator; Mach-Zehnder modulator

\section{Introduction}

The Si photonics platform enables the fabrication of dense photonic integrated circuits and reduces their production cost, thanks to the strong optical confinement of high-index-contrast Si devices and the utilization of the matured complementary metal-oxide-semiconductor (CMOS) process, respectively. It is well suited for the development of components and subsystems for optical interconnects in data centers and high-performance computers. For these cases, optical modulators are key [1-6] and a wide working spectrum and wide working temperature range are particularly required in optical interconnects, in addition to small size, low loss, low drive voltage and high speed.

Previous studies on Si modulators have mainly used a phase shift generated by carrier plasma dispersion in p-n-doped rib waveguides. Resonator-type modulators such as microring devices often show good performance with a small size, but the working spectrum is very limited and cannot operate stably without complicated thermal stabilizations [7-9]. Therefore, most studies have employed Mach-Zehnder (MZ) devices, enabling a wide working spectrum and, hence, operating over a wide temperature range without thermal stabilizations when they use a symmetric circuit. However, MZ circuits typically need a long phase shifter length of several millimeters to obtain a sufficient extinction ratio $(E R)$ [1-6], resulting in high power consumption. To overcome this constraint, we have developed photonic crystal waveguide (PCW) MZ modulators. They are constructed via a CMOS process, with a phase shifter length $L$ significantly reduced by the slow-light effect [10-15]. For 
slow light with a low group velocity $v_{\mathrm{g}}$, the slowdown factor is usually expressed by the group index $n_{\mathrm{g}} \equiv c / v_{\mathrm{g}}$, where $c$ is the light velocity in a vacuum. Values of $n_{\mathrm{g}}$ are observed to be between 3 and 4 for Si rib waveguides, while Si PCWs easily produce $n_{\mathrm{g}}>20$ [10]. In a simplified model, the phase shift $\Delta \phi$ in a waveguide with an equivalent modal index change induced by carrier plasma dispersion, $\Delta n_{\text {eq }}$, is expressed as $[15,16]$

$$
\Delta \phi=k_{0} n_{\mathrm{g}} \frac{\Delta n_{\mathrm{eq}}}{n_{\mathrm{eq}}} \zeta L, \zeta \equiv \frac{n_{\mathrm{eq}}}{\omega_{\mathrm{b}}} \frac{d \omega_{\mathrm{b}}}{d n_{\mathrm{eq}}} \approx 1
$$

where $k_{0}=2 \pi / \lambda$ is the wave number in a vacuum and $\omega_{\mathrm{b}}$ is the photonic band frequency. As $\Delta \phi$ is proportional to $n_{\mathrm{g}}$, the same value for $\Delta \phi$ can be expected even for smaller $L$ and $\Delta n_{\mathrm{eq}}$ by employing a large $n_{\mathrm{g}}$. Furthermore, we developed lattice-shifted PCWs (LSPCWs) that generate low-dispersion (LD) slow light, showing an almost constant $n_{\mathrm{g}}$ over the wide wavelength range of 10-20 $\mathrm{nm}$ for transverse-electric (TE) polarization [10]. In our recent study, we observed eye opening at some selected wavelengths and the unchanged 10 Gbps operation was confirmed for a wavelength bandwidth of $16.9 \mathrm{~nm}$ at wavelength $\lambda \sim 1550 \mathrm{~nm}$ and a temperature range of $19-124{ }^{\circ} \mathrm{C}$ in a device with $L=90 \mu \mathrm{m}$ [11]. Moreover, an interleaved p-n junction was employed, which increases the overlap of a depletion region with the slow-light mode and thus increases $\Delta n_{\text {eq }}$. This succeeded in the $25 \mathrm{Gbps}$ modulation in a device with $L=200 \mu \mathrm{m}$ with a drive voltage of $V_{\mathrm{pp}}=1.75 \mathrm{~V}$ (on the panel of pulse pattern generator, PPG) [15]. In this study, we observed an $E R$ of $3 \mathrm{~dB}$, although the excess modulation loss $(M L)$, which usually helps to increase the $E R$, was moderately suppressed to $0.8 \mathrm{~dB}$.

A concern in using such LD slow light in practical modulators is the small fluctuations of $n_{\mathrm{g}}$ in a working spectrum. These fluctuations are considered to be primarily caused by the reflection at the ends of the LSPCW and may be suppressed by technical improvements. However, for the fundamental improvement of the fabrication accuracy, a more advanced CMOS process is required, which increases the production cost. It is important to estimate how severely modulation characteristics reflect fluctuations in $n_{\mathrm{g}}$ and thus modify requirements accordingly. In this study, we investigated modulation characteristics over the wide working spectrum. We measured the frequency response, $E R$, and bit-error rate $(B E R)$ in detail and evaluated the relation with $n_{\mathrm{g}}$, and revealed the condition that satisfies requirements for optical interconnects.

\section{Device}

Figure 1a shows the schematic of the Si LSPCW MZ modulator with the interleaved p-n junction. The details of the structure, theoretical modal behaviors, and fundamental modulation characteristics can be seen in Reference [15]. Third-row-shifted LSPCWs with a central p-n junction and $\mathrm{SiO}_{2}$ cladding as phase shifters were employed; these exhibit typical LD slow light. The phase shifter length was fixed at $L=200 \mu \mathrm{m}$ and parameters of the LSPCW were $a$ (lattice constant) $=400 \mathrm{~nm}$, $2 r$ (hole diameter) $=220 \mathrm{~nm}$, and $s$ (lattice shift) $=95 \mathrm{~nm}$. An interleaved p-n junction with a comb period of $w_{\mathrm{x}}=300 \mathrm{~nm}$ and a comb depth of $w_{\mathrm{y}}=600 \mathrm{~nm}$ was used. The $\mathrm{p}$ - and n-type doping concentrations were given as $N_{\mathrm{A}}=9.5 \times 10^{17} \mathrm{~cm}^{-3}$ and $N_{\mathrm{D}}=5.7 \times 10^{17} \mathrm{~cm}^{-3}$, respectively, and the $\mathrm{p}^{+}$and $\mathrm{n}^{+}$doping concentrations for electrical contacts were $N_{\mathrm{A}}^{+}=1.0 \times 10^{19} \mathrm{~cm}^{-3}$ and $N_{\mathrm{D}}{ }^{+}=1.0 \times 10^{19} \mathrm{~cm}^{-3}$, respectively. To avoid the strong free carrier absorption in heavily doped regions, a distance of $\sim 4 \mu \mathrm{m}$ across the p-n junction was set. As radio frequency (RF) electrodes of the phase shifters, coplanar waveguides consisting of an Al electrode for signal (S) and two Al electrodes for ground $(\mathrm{G})$ were employed. No termination resistors were used, to simplify the device layout. In addition, $36.5 \mu \mathrm{m}$ TiN heaters were integrated inside the $\mathrm{SiO}_{2}$ cladding above the same LSPCWs, and were used for setting the initial phase of each $\mathrm{MZ}$ arm. The total footprint of the device excluding the input and output waveguides was $\sim 400 \times 500 \mu \mathrm{m}^{2}$, but they could be reduced to $\sim 50 \times 250 \mu \mathrm{m}^{2}$ if we shrink the electrodes and neglect the RF pads. 


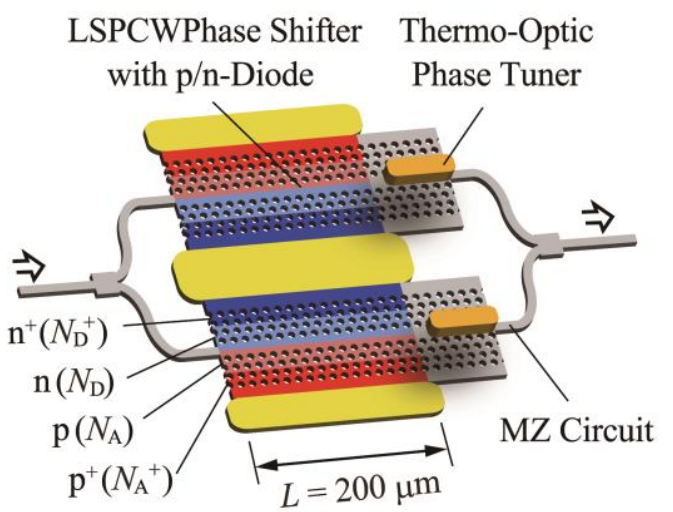

(a)

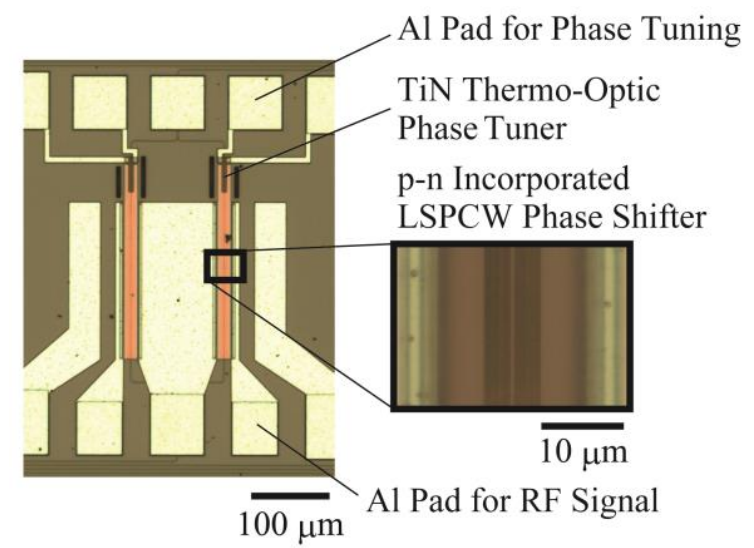

(b)

Figure 1. Si LSPCW MZ modulator. (a) Schematic. Interleaved p-n junction was employed but not shown in this figure; (b) Microscope image of fabricated device.

The devices were constructed on a $200 \mathrm{~mm}$ silicon-on-insulator (SOI) wafer, using a $180 \mathrm{~nm}$ CMOS process at the Institute of Micro-Electronics foundry service with a $\mathrm{KrF}$ excimer laser exposure at $\lambda=248 \mathrm{~nm}$. The original thickness of the Si slab in the SOI wafer was $220 \mathrm{~nm}$, but was subsequently thinned to $210 \mathrm{~nm}$ by thermal oxidation. The thickness of the $\mathrm{SiO}_{2} \mathrm{BOX}$ layer was $2 \mu \mathrm{m}$. Fourteen mask layers were used: two layers for the waveguides, four layers for the p-n junctions, two layers for the heaters and thermal isolation trench, four layers for RF and DC electrodes, contact holes, and contact pads, and two layers for the formation of waveguide end facets with spot size converters (SSC). For the waveguide layers, phase-shift masks were applied to improve the resolution to approximately $100 \mathrm{~nm}$. Figure $1 \mathrm{~b}$ shows the microscope image of the fabricated device.

First, we measured the fundamental slow-light characteristics of the device. Using objective lenses or compact lens modules, we input light from and output light to single mode fibers with the SSC consisting of $\mathrm{SiO}_{2}$ waveguides and $\mathrm{Si}$ inverse tapers located at the chip facets. Figure 2a shows the transmission spectrum normalized by the transmission in a $\mathrm{Si}$ wire waveguide of the same length. The device exhibits a flat transmission band at $\lambda=1550-1570 \mathrm{~nm}$ with a maximum transmission of $-5 \mathrm{~dB}$. This excess loss came from the two $1 \times 2$ couplers in the MZ circuit $(<0.5 \mathrm{~dB}$ in total), the propagation loss in the LSPCW $(\sim 3 \mathrm{~dB})$, and the coupling loss between the LSPCW and Si wire $(\sim 1.5 \mathrm{~dB}$ in total). More than $60 \%$ of the LSPCW loss is due to the free-carrier absorption, which is expected to be reduced by optimizing the doping. Therefore, the excess loss is expected to fall by $\sim 3 \mathrm{~dB}$ in future studies. Since the Si wire for the optical wiring had a $4 \mathrm{~dB} / \mathrm{cm}$ propagation loss and a $<0.1 \mathrm{~dB}$ loss at $90^{\circ}$ bends, which corresponds to a $2 \mathrm{~dB}$ loss for $\sim 4.5-\mathrm{mm}$-long wiring with six bends, the total on-chip loss of the device was $7 \mathrm{~dB}$. Figure $2 \mathrm{~b}$ shows the $n_{\mathrm{g}}$ spectrum measured by the modulation phase shift method. The device exhibited the LD band with $n_{\mathrm{g}} \approx 20$ and bandwidth $\Delta \lambda \approx 20 \mathrm{~nm}$. We have reported higher $n_{\mathrm{g}}$ of $50-60$ by modifying the lattice shift. However, in that case, the working spectrum was narrowed to less than $10 \mathrm{~nm}$ due to the constraint of the $n_{\mathrm{g}}$-bandwidth product [10], and the strong back-scattering in the PCW, with some disordering, produced irregular oscillations. In this study, since we set $n_{\mathrm{g}}$ to be moderately low, the $n_{\mathrm{g}}$ spectrum was sufficiently wide and looks to be almost flat with no irregular oscillations. The $\pm 10 \%$ variation of $n_{\mathrm{g}}$ observed in the $n_{\mathrm{g}}$ spectrum is considered to be due to the Fabry-Perot resonance in the LSPCW [17]. 


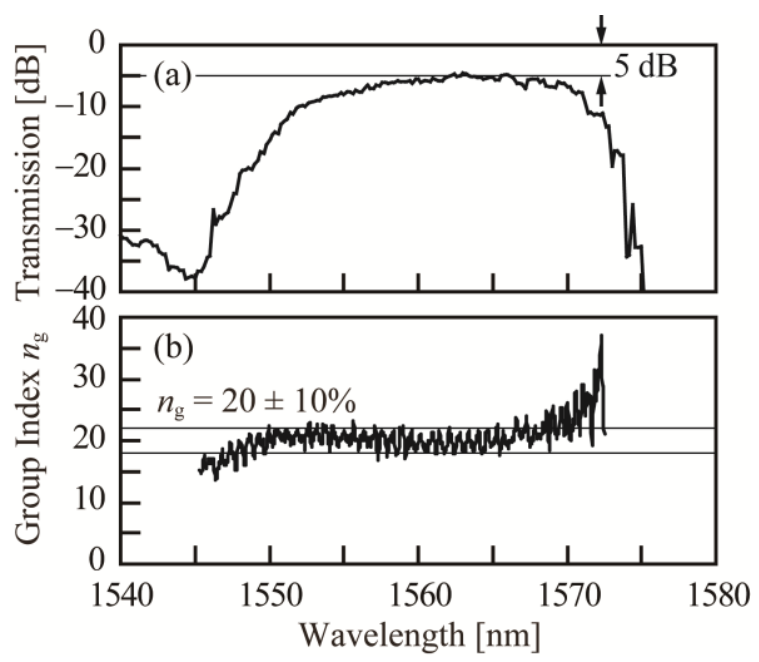

Figure 2. Slow-light characteristics of fabricated device. (a) Transmission spectrum; (b) $n_{\mathrm{g}}$ spectrum.

\section{Frequency Response}

The frequency response was measured using a vector network analyzer (VNA, Anritsu 37269E-R, Atsugi, Japan). Small sinusoidal signals from the VNA were applied to the RF electrode of a single $\mathrm{MZ}$ arm through an RF probe (typical insertion loss is $0.8 \mathrm{~dB}$ ), and the modulated signals were then returned to the VNA through an erbium-doped fiber amplifier (EDFA, Alnair Labs CPA-100-CL, Shinagawa, Japan), a band-pass filter (BPF, Alnair Labs CVF-220-CL, Shinagawa, Japan) and an opto-electronic (OE) converter (Anritsu MN4765, Atsugi, Japan). Figure 3 shows $S_{11}$ for the RF signals reflected to the output port of the VNA and $S_{21}$ for the modulated optical signals that were input into the VNA. As previously mentioned, the device did not have termination resistors, and thus $S_{11}$ becomes $0 \mathrm{~dB}$ at low frequencies. At high frequencies, on the other hand, the RF signals tend to attenuate during the transmission due to the parasitic resistance at the $\mathrm{p}-\mathrm{n}$ junction, which decreases $S_{11}$ gradually. The dip appearing at $19 \mathrm{GHz}$ is considered to be caused by the excitation of the coupled slotline mode. This will be suppressed by symmetrizing the GSG RF electrode, which was designed asymmetrically in this fabrication, and the connection between the two $G$ electrodes $[4,18]$. In any case, the reflection of the RF signals generates a voltage on the electrode two times greater than the set value at the PPG at low frequencies, 1.7 times greater at $12 \mathrm{GHz}$ exhibiting $S_{11}=-3 \mathrm{~dB}$, and 1.4-1.5 times greater at $15 \mathrm{GHz}$ exhibiting $S_{11}=-6 \mathrm{~dB}$. This enhances the total modulation efficiency while relatively degrading the high-frequency response.

For the electro-optic (EO) $S_{21}$ measurement, a TE-polarized laser light at $\lambda=1554 \mathrm{~nm}$ was coupled to the SSC through a polarization-maintaining single mode fiber with a lens module. The coupling loss was $3.5 \mathrm{~dB}$, including a $1 \mathrm{~dB}$ loss inside the lens module ( $7 \mathrm{~dB}$ in total for the input and output). The total insertion loss from fiber to fiber was approximately $14 \mathrm{~dB}$ including a $7 \mathrm{~dB}$ on-chip loss. Furthermore, the total loss from the laser source to the output fiber was $21 \mathrm{~dB}$, including losses at the polarization controller and some junctions between fibers. Therefore, the laser power was raised to $16.5 \mathrm{dBm}$ and $S_{21}$ was measured. The cutoff frequency $f_{3 \mathrm{~dB}}$ was $17 \mathrm{GHz}$, which is sufficient for the $25 \mathrm{Gbps}$ operation. In general, $f_{3 \mathrm{~dB}}$ is constrained by the $R C$ time constant. We have reported the calculation of the resistance $R$ and capacitance $C$ at the p-n junction using the commercial simulator Lumerical DEVICE in Reference [15]. Modeling the Si layer sandwiched by the $\mathrm{SiO}_{2} \mathrm{BOX}$ layer and top $\mathrm{SiO}_{2}$ cladding (both insulators), they were calculated to be $96 \Omega$ (146 $\Omega$ including $50 \Omega$ internal resistance in the PPG connected in series with the $R$ ) and $\sim 60 \mathrm{fF}$ at $V_{\mathrm{b}}=-2.2 \times 2 \mathrm{~V}$, respectively, for the current design. Their product gives $f_{3 \mathrm{~dB}}=18 \mathrm{GHz}$, which almost agrees with the measured value. 
(a)

(b)
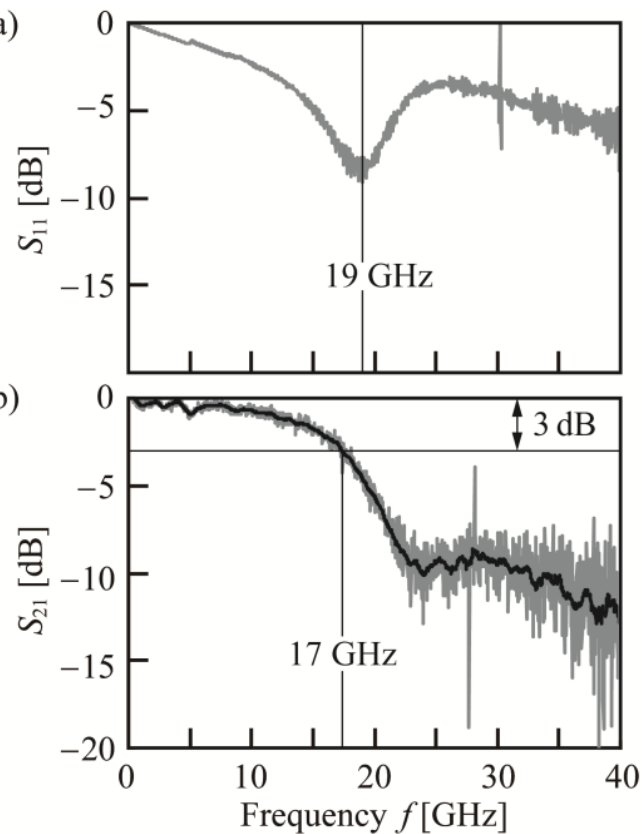

Figure 3. Measured $S$ parameters: (a) $S_{11}$; (b) EO $S_{21}$ at $\lambda=1554 \mathrm{~nm}$. Gray line shows raw data. Black line shows averaged values within each $\sim 0.5 \mathrm{GHz}$ range.

The $f_{3 \mathrm{~dB}}$ could be constrained by the phase mismatch between the slow light and RF signals. The decreased ratio of the modulation depth, $\eta(f)$, is expressed as

$$
\eta(f)=\frac{1}{L} \int_{0}^{L} \cos \left\{\left(n_{\mathrm{g}}-n_{\mathrm{RF}}\right) \frac{2 \pi f z}{c}+\theta_{0}\right\} d z
$$

where $n_{\mathrm{RF}}$ is the refractive index for RF signals, $c$ is the light velocity in a vacuum, and $\theta_{0}$ is the initial phase mismatch. EO $S_{21}$ is expressed using electro-electronic (EE) $S_{21}$ for RF signals as

$$
\mathrm{EO} S_{21}=\eta(f) \cdot \operatorname{EE} S_{21}
$$

For typical traveling-wave modulators, a small phase mismatch is compensated by optimizing the design. In slow-light modulators, on the other hand, this issue is more complicated due to the large differences between group velocities. Let us assume that $f=12.5 \mathrm{GHz}$ is a critical frequency for $25 \mathrm{Gbps}$ modulation. Then, for $n_{\mathrm{g}}=20, n_{\mathrm{RF}}=2$ and $L=200 \mu \mathrm{m}, \eta(f)$ is calculated to be higher than 0.95 when $\theta_{0}$ is optimized. Thus, the phase mismatch issue is not so severe. However, if either $n_{\mathrm{g}}$, $L$ or $f$ is larger than their present values, it will become a limiting factor to the frequency response; $\eta(f)$ will be less than 0.9 when $n_{\mathrm{g}} \geqslant 32, L \geqslant 340 \mu \mathrm{m}$ or $f \geqslant 22 \mathrm{GHz}$.

We measured the frequency response spectrum $R(f, \lambda)$ by changing $\lambda$ in steps of $0.02 \mathrm{~nm}$ in the LD band within the range $\lambda=1550-1558 \mathrm{~nm}$. Figure 4 shows the measured results as a color plot, as well as the measured $n_{\mathrm{g}}$ spectrum and calculated $f_{3 \mathrm{~dB}}$ spectrum from Equations (2) and (3). $R(f, \lambda)$ exhibits fluctuations, which are considered to reflect the $n_{\mathrm{g}}$ spectrum, although they do not agree completely. The $n_{\mathrm{g}}$ spectrum shows a relatively large and slow oscillation with an averaged period of $0.7 \mathrm{~nm}$, and a small and fast oscillation with an averaged period smaller than $0.2 \mathrm{~nm}$. This large and slow oscillation roughly corresponds to the fluctuations in $R(f, \lambda)$ at $f>20 \mathrm{GHz}$, and therefore may correspond to the effect of the phase mismatch at higher $f$. On the other hand, $f_{3 \mathrm{~dB}}$ depicted by the boundary between red and yellow regions only exhibits the small and fast fluctuation, lying between 15 and $20 \mathrm{GHz}$, with the exception of three wavelengths that show $f_{3 \mathrm{~dB}}=12-13 \mathrm{GHz}$. Thus, the frequency response of this device satisfies the requirement for the 25 Gbps operation over the wide wavelength range. 


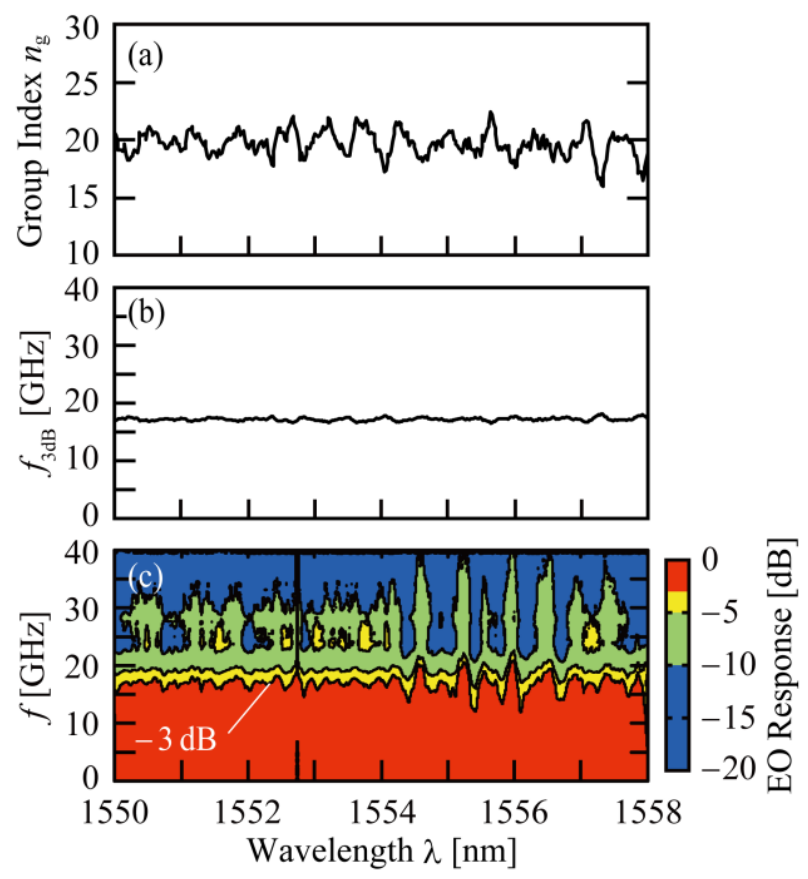

Figure 4. Fluctuations in frequency response over the wide wavelength range: (a) $n_{\mathrm{g}}$ spectrum; (b) $f_{3 \mathrm{~dB}}$ spectrum calculated from Equations (2) and (3) with the measured $n_{\mathrm{g}}$ and EE $S_{21}$ estimated with $R=96 \Omega$ and $C=50 \mathrm{fF}$; (c) Frequency response spectrum $R(f, \lambda)$ obtained from the averaged response at each $\lambda$, such as those represented by the black line in Figure $3 \mathrm{~b}$. The $f_{3 \mathrm{~dB}}$ is shown by the boundary between red and yellow regions. The fluctuation in the measurement setup was smaller than $1 \mathrm{GHz}$.

\section{Signal Modulation}

The 25 Gbps signal modulation was investigated over the wide wavelength range, using the non-return-to-zero (NRZ) pseudo-random-bit sequence (PRBS). Although the voltages from PPG (Anritsu MP1800A, Atsugi, Japan) were set as $V_{\mathrm{b}} \approx-1 \mathrm{~V}$ and $V_{\mathrm{pp}}=2.0 \mathrm{~V}$, actual applied voltages should be modified by the open-ended electrodes, as mentioned above. The operation was first measured at $\lambda=1561 \mathrm{~nm}$. Since the device and measurement setup had losses as outlined above, the output light from the device was amplified using EDFA and BPF, and then received and analyzed by an optical sampling oscilloscope (Keysight 86100C, 86109A, Santa Rosa, CA, USA). Figure 5a shows the eye pattern when the initial phase of the $M Z$ arms was adjusted so that $E R \approx 3 \mathrm{~dB}$ was obtained. The open eye was observed with $M L=0.5 \mathrm{~dB}$, which is comparable to that in a previous study [15]. Figure $5 \mathrm{~b}$ shows a clearer open eye with $E R=3.9 \mathrm{~dB}$ when the $M L$ was slightly increased to $1 \mathrm{~dB}$.

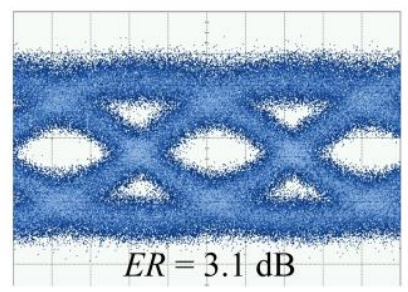

(a)

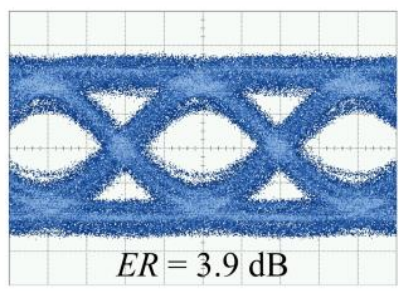

(b)

Figure 5. The $25 \mathrm{Gbps}$ eye patterns of modulated light: (a) $M L=0.5 \mathrm{~dB}$ and (b) $1.0 \mathrm{~dB}$ with PRBS lengths of $2^{31}-1$ and $2^{7}-1$, respectively. 
Next, ER was repeatedly measured, changing the wavelength in steps of $0.5 \mathrm{~nm}$ between the range $\lambda=1549.5-1565.5 \mathrm{~nm}$. The initial phase was adjusted such that the output intensity with no $V_{\mathrm{pp}}$ was decreased to $2 \mathrm{~dB}$ lower than the maximum, thus giving $M L=2-E R / 2$. This is almost the same condition as that in Figure 5. Figure 6 shows the spectra of $E R$ and $n_{\mathrm{g}}$. Although they do not agree completely, they exhibit similar patterns. $E R$ ranges between 2 and $3 \mathrm{~dB}$ and fluctuates by $\sim 1 \mathrm{~dB}$. Furthermore, $E R$ and $B E R$ were measured, within the range $\lambda=1558-1560 \mathrm{~nm}$ in steps of $0.2 \mathrm{~nm}$. For the BER measurement, amplified light was detected by an OE converter (Agilent 11982A) after passing through a variable optical attenuator, where the electrical signal was incident on an error detector module (Anritsu MU183040B) after removing the DC components. Voltages from the PPG and initial phase were set similarly to those presented in Figure 6. Figure 7a shows BER measured as a function of the received powers $P_{\mathrm{r}}$ at the $\mathrm{OE}$ converter. The error-free operation of $B E R<10^{-11}$ was confirmed. Figure 7 compares the $B E R$ and $E R$ spectra measured in steps of $0.2 \mathrm{~nm}$ in the range of $\lambda=1558-1560 \mathrm{~nm}$. ER fluctuated by $1 \mathrm{~dB}$, similarly to that in Figure 6 . In other words, the $B E R$ finally reflects the fluctuations in $n_{\mathrm{g}}$ through those in the ER. This indicates that a margin larger than $1 \mathrm{~dB}$ is necessary in ER for a stable, error-free operation. For optical interconnects, ER is required to be larger than $3 \mathrm{~dB}$, although it depends on the specifications of the transmitter and receiver. However, for the modulator described in this study, ER should be greater than $4 \mathrm{~dB}$, including the margin. This is achieved by increasing the $M L$ by $0.5 \mathrm{~dB}$ and the loss is compensated by increasing the output power from the light source or reducing the passive losses.

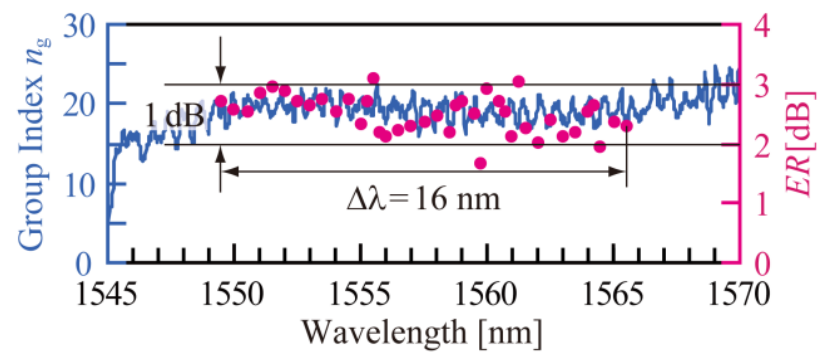

Figure 6. Spectra of $n_{\mathrm{g}}$ and $E R$ measured at $25 \mathrm{Gbps}$. Operating condition was similar to that for Figure 5 .

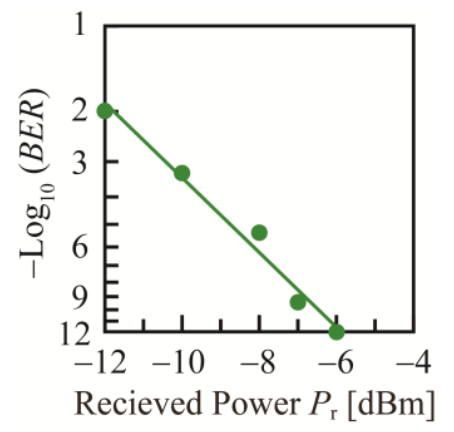

(a)

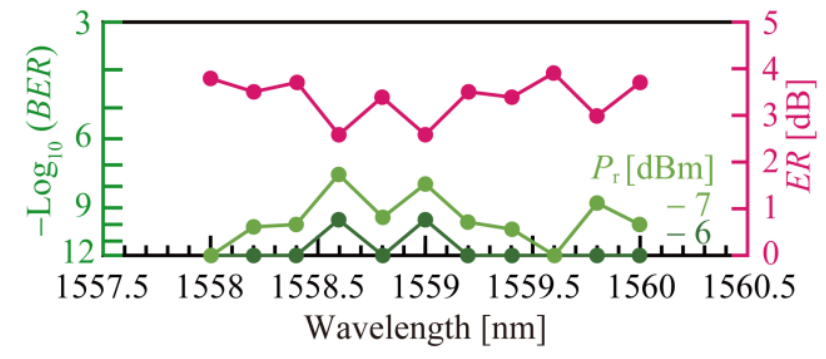

(b)

Figure 7. $B E R$ characteristics at 25 Gbps for $2^{7}-1$ bit NRZ-PRBS signals: (a) $B E R$ at $\lambda=1559.6 \mathrm{~nm}$; (b) $B E R$ and $E R$ at $\lambda=1558-1560 \mathrm{~nm}$.

\section{Slow-Light Enhancement}

Thus far, the modulation characteristics have been presented with a fixed $n_{\mathrm{g}} \approx 20$. In this section, the characteristics for a varied $n_{\mathrm{g}}$ are presented. For a $\mathrm{SiO}_{2}$-cladded PCW, without a lattice shift, $n_{\mathrm{g}}$ increases as $\lambda$ increases towards the photonic band edge. Since this increase is smaller than that in air-bridge structures which have been extensively studied in the past, this allows for a wide scan 
of $n_{\mathrm{g}}$ and the comprehensive investigation of the slow-light enhancement, by only changing $\lambda$. Here, the PCW was designed with specifications of $a=400 \mathrm{~nm}, 2 r=200 \mathrm{~nm}$, and $s=0 \mathrm{~nm}$. Due to different fabrication lots, doping concentrations were lower than those given above, i.e., $N_{\mathrm{A}}=4.8 \times 10^{17} \mathrm{~cm}^{-3}$ and $N_{\mathrm{D}}=4.8 \times 10^{17} \mathrm{~cm}^{-3}$, and the p-n junction profile was linear. Therefore, the modulation efficiency should be lower than that in the device described in the previous sections. The absolute value of the efficiency was neglected, while focusing on the relative change of the modulation characteristics with $n_{\mathrm{g}}$.

Figure 8 presents the measured $n_{\mathrm{g}}$ spectrum, with the $f_{3 \mathrm{~dB}}$ spectrum calculated in the same way as that for Figure $4 \mathrm{~b}$, and measured $R(f, \lambda)$ at $\lambda=1534-1540 \mathrm{~nm}$. It is shown that $n_{\mathrm{g}}$ changes widely from 20 to 60 within this range. The calculated $f_{3 \mathrm{~dB}}$ decreases with the increasing $n_{\mathrm{g}}$, showing the influence of the phase mismatch. The $f_{3 \mathrm{~dB}}$ estimated from the measured $R(f, \lambda)$ was $10-20 \mathrm{GHz}$ at $n_{\mathrm{g}} \approx 20$, but then fell to less than $10 \mathrm{GHz}$ for higher $n_{\mathrm{g}}$, which almost agrees with the calculated one. Thus, some solution to suppress the phase mismatch is necessary when $n_{\mathrm{g}}$ is increased to enhance $\Delta \phi$. Solutions need to slow down the RF signals and/or to shift back the phase of the RF signals inside the electrode. For the former, a slow-wave electrode consisting of a loaded transmission line has previously been studied [6,19-22]. However, their slow-down factors are too small to compensate the phase mismatch. For the latter, it is possible to add a delay line, such as a meander line; however, this will increase the device footprint as its length will be in the order of millimeters. Furthermore, the fluctuations in $f_{3 \mathrm{~dB}}$ in Figure 8 become particularly large at $n_{\mathrm{g}}>40$, possibly caused by the oscillation of $n_{\mathrm{g}}$. As previously mentioned, a large $n_{\mathrm{g}}$ generates strong back-scattering and, in particular, enlarges the spectral oscillation. A more advanced CMOS process is necessary to completely improve this situation, which may be costly. Alternatively, $n_{\mathrm{g}}$ can be set to no higher than 40 if the current CMOS process is used, with the application of the slow light to the modulator.

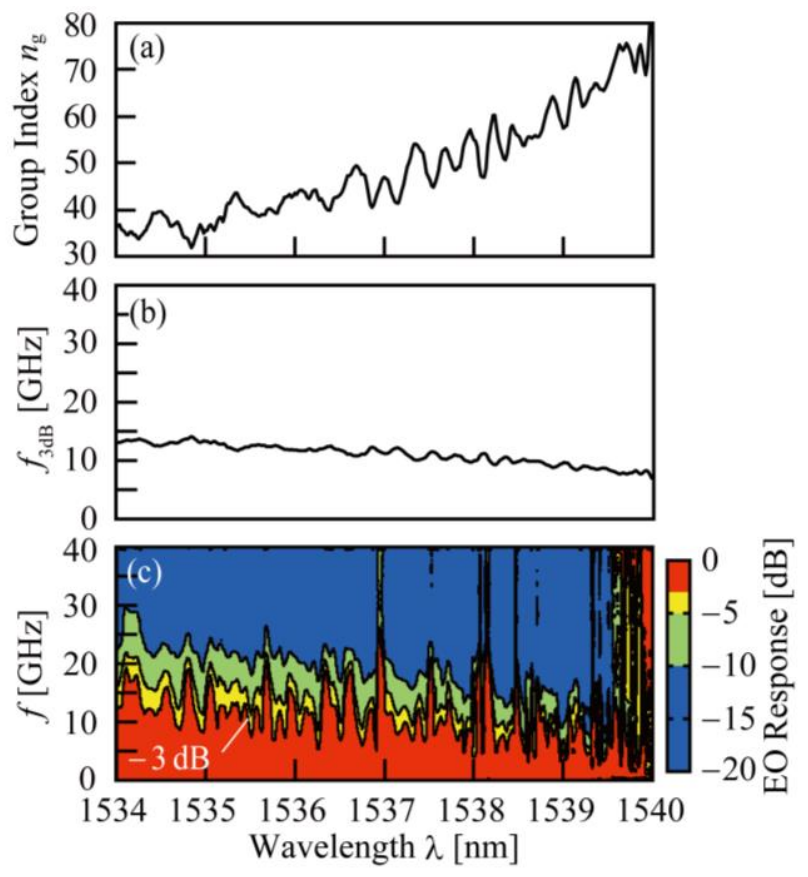

Figure 8. Characteristics of PCW device without lattice shifts in the wide wavelength range: (a) $n_{\mathrm{g}}$ spectrum; (b) $f_{3 \mathrm{~dB}}$ spectrum calculated from Equations (2) and (3) with the measured $n_{\mathrm{g}}$ and EE $S_{21}$ estimated with $R=96 \Omega$ and $C=50 \mathrm{fF}$; (c) Frequency response spectrum $R(f, \lambda)$.

Figure 9 shows the result of the signal modulations. Although the voltages are the same as for Figure 5, the initial phase was set such that the transmission with no $V_{\mathrm{pp}}$ was reduced to $9 \mathrm{~dB}$ lower than the maximum, resulting in a large $M L$, and, hence, compensating for the low modulation 
efficiency. The $E R$ was $2.5 \mathrm{~dB}$ at $n_{\mathrm{g}}=25$, but increased to $8.1 \mathrm{~dB}$ at $n_{\mathrm{g}}=64$. Figure 10 shows $\Delta \phi$ estimated from the $E R$ as a function of $n_{\mathrm{g}}$. As expected from Equation (1), $\Delta \phi$ is almost proportional to $n_{\mathrm{g}}$, and therefore confirms the slow-light enhancement in the modulation efficiency. Equation (1) also indicates that $\Delta \phi$ depends on $\Delta n_{\mathrm{eq}}$, which is determined by the overlap between the guided mode and the depletion region of the p-n junction. If the distribution of the slow-light mode changes with $n_{\mathrm{g}}, \Delta n_{\text {eq }}$ would also change. Inversely speaking, the proportional behaviors between $\Delta \phi$ and $n_{\mathrm{g}}$ suggest that the distribution is almost unchanged or is less severe for the modulator performance in this range. Equations (2) and (3) and the results in Figure 8 also suggest that such large $n_{\mathrm{g}}$ makes the phase mismatch more severe and decreases $\Delta \phi$. However, it is not apparent in Figure 10 because the slow-light enhancement in Equation (1) might be more dominant for the 25 Gbps PRBS signals whose major frequency components are lying at $<12.5 \mathrm{GHz}$.

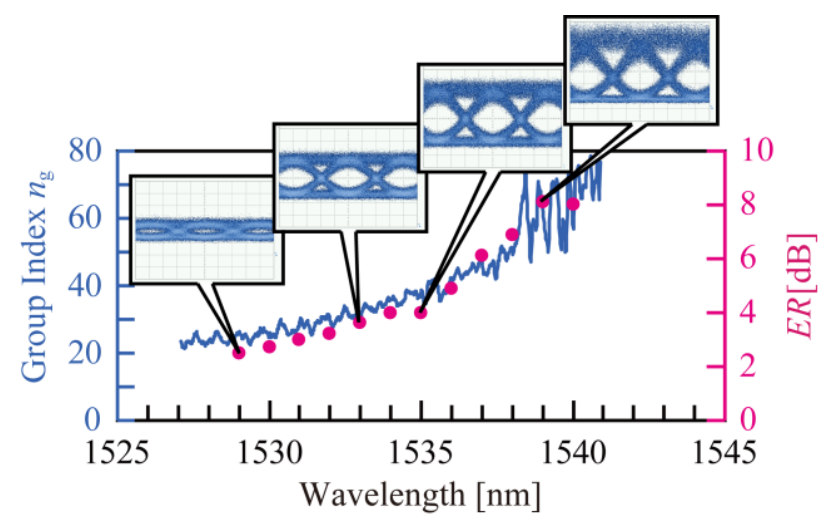

Figure 9. The $n_{\mathrm{g}}$ spectrum of PCW device without lattice shifts and ER at 25 Gbps modulation. Set voltages were the same as those for Figure 5.

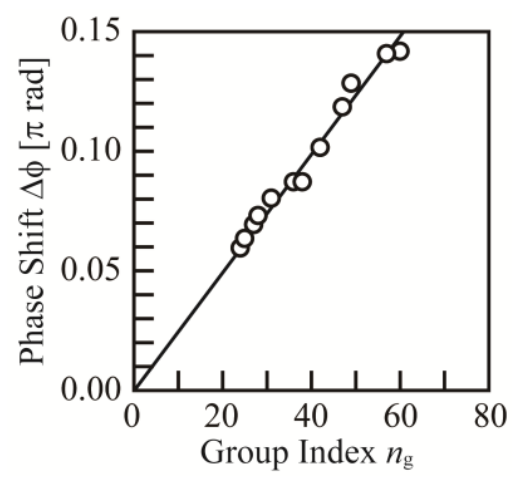

Figure 10. The $\Delta \phi$ estimated from $E R$ as a function of $n_{\mathrm{g}}$

\section{Conclusions}

Si lattice-shifted photonic crystal slow-light modulators were constructed using a $180 \mathrm{~nm}$ CMOS process, with a phase shifter length as short as $200 \mu \mathrm{m}$. The frequency response, $E R$, and $B E R$ were measured in wide wavelength ranges, with fluctuations observed, perhaps due to the oscillation in the group index $n_{\mathrm{g}}$ spectrum. The device with $n_{\mathrm{g}} \approx 20$ showed a wide working spectrum of $20 \mathrm{~nm}$ and a sufficient frequency response for the $25 \mathrm{Gbps}$ modulation. A higher $n_{\mathrm{g}}$ up to 60 is available by modifying the lattice shift but the working spectrum is comparably narrowed, which is the major constraint of the slow-light effect. Even for the moderately low $n_{\mathrm{g}} \approx 20$, the $\pm 10 \%$ fluctuation was observed in the $n_{\mathrm{g}}$ spectrum, which was reflected in $E R$ and $B E R$. Therefore, a margin larger than $1 \mathrm{~dB}$ is necessary in $E R$ for stable, error-free operation, which can be achieved by adding a $0.5 \mathrm{~dB}$ modulation loss, comparably increasing the light source power and/or reducing passive losses. The ER 
was simply increased by increasing $n_{\mathrm{g}}$; however, the frequency response was degraded simultaneously. This degradation was considered to be due to the phase mismatch between slow light and RF signals, which will be more problematic beyond 25 Gbps. Results suggest that $n_{\mathrm{g}}$ should be set to less than 40 to avoid this degradation and avoid irregularly large oscillations in $E R$, which is particularly enhanced by slow light.

Acknowledgments: This work was partly supported by the New Energy and Industrial Technology Development Organization (NEDO), Japan.

Author Contributions: Yosuke Hinakura, Yosuke Terada and Toshihiko Baba conceived and designed the experiments; Yosuke Terada, Takuya Tamura and Toshihiko Baba designed the devices; Yosuke Hinakura and Yosuke Terada performed the experiments; Yosuke Hinakura analyzed the data; Toshihiko Baba contributed experiment systems, analysis tools; Yosuke Hinakura and Toshihiko Baba wrote the paper.

Conflicts of Interest: The authors declare no conflict of interest.

\section{References}

1. Reed, G.T.; Mashanovich, G.; Gardes, F.Y.; Thomson, D.J. Silicon optical modulators. Nat. Photonics 2010, 4, 518-526. [CrossRef]

2. Thomson, D.J.; Gardes, F.Y.; Fedeli, J.M.; Zlatanovic, S.; Hu, Y.; Kuo, B.P.P.; Myslivets, E.; Alic, N.; Radic, S.; Mashanovich, G.Z.; et al. 50-Gb/s silicon optical modulator. IEEE Photonics Technol. Lett. 2012, 24, 234-236. [CrossRef]

3. Yang, L.; Ding, J. High-speed silicon Mach-Zehnder optical modulator with large optical bandwidth. J. Lightwave Technol. 2014, 32, 966-970. [CrossRef]

4. Xu, H.; Li, X.; Xiao, X.; Li, Z.; Yu, Y.; Yu, J. Demonstration and characterization of high-speed silicon depletion-mode Mach-Zehnder modulators. IEEE J. Sel. Top. Quantum Electron. 2014, 20, 23-32. [CrossRef]

5. Akiyama, S.; Imai, M.; Baba, T.; Akagawa, T.; Hirayama, N.; Noguchi, Y.; Seki, M.; Koshino, K.; Toyama, M.; Horikawa, T.; et al. Compact PIN-diode-based silicon modulator using side-wall-grating waveguide. IEEE J. Sel. Top. Quantum Electron. 2013, 19, 74-84. [CrossRef]

6. Ding, R.; Liu, Y.; Ma, Y.; Yang, Y.; Li, Q.; Lim, A.E.J.; Lo, G.Q.; Bergman, K.; Baehr-Jones, T.; Hochberg, M. High-speed silicon modulator with slow-wave electrodes and fully independent differential drive. J. Lightwave Technol. 2014, 32, 2240-2247. [CrossRef]

7. Timurdogan, E.; Sorace-Agaskar, C.M.; Sun, J.; Hosseini, E.S.; Biberman, A.; Watts, M.R. An ultralow power athermal silicon modulator. Nat. Commun. 2014, 5, 4008. [CrossRef] [PubMed]

8. Zheng, X.; Chang, E.; Amberg, P.; Shubin, I.; Lexau, J.; Liu, F.; Thacker, H.; Djordjevic, S.S.; Lin, S.; Luo, Y.; et al . A high-speed, tunable silicon Photonic ring modulator integrated with ultra-efficient active wavelength control. Opt. Express 2014, 22, 12628-12633. [CrossRef] [PubMed]

9. Sun, C.; Wade, M.T.; Lee, Y.; Orcutt, J.S.; Alloatti, L.; Georgas, M.S.; Waterman, A.S.; Shainline, J.M.; Avizienis, R.R.; Lin, S.; et al. Single-chip microprocessor that communicates directly using light. Nature 2015, 528, 534-538. [CrossRef] [PubMed]

10. Tamura, T.; Kondo, K.; Terada, Y.; Hinakura, Y.; Ishikura, N.; Baba, T. Silica-Clad Silicon Photonic Crystal Waveguides for Wideband Dispersion-Free Slow Light. J. Lightwave Technol. 2015, 33, 3034-3040. [CrossRef]

11. Nguyen, H.C.; Sakai, Y.; Shinkawa, M.; Ishikura, N.; Baba, T. 10 Gb/s operation of photonic crystal silicon optical modulators. Opt. Express 2011, 19, 13000-13007. [CrossRef] [PubMed]

12. Nguyen, H.C.; Sakai, Y.; Shinkawa, M.; Ishikura, N.; Baba, T. Photonic crystal silicon optical modulators: Carrier-injection and depletion at $10 \mathrm{~Gb} / \mathrm{s}$. IEEE J. Quantum Electron. 2012, 48, 210-220. [CrossRef]

13. Nguyen, H.C.; Hashimoto, S.; Shinkawa, M.; Baba, T. Compact and fast photonic crystal silicon optical modulators. Opt. Express 2012, 20, 22465-22474. [CrossRef] [PubMed]

14. Nguyen, H.C.; Yazawa, N.; Hashimoto, S.; Otsuka, S.; Baba, T. Sub-100 $\mu \mathrm{m}$ photonic crystal Si optical modulators: Spectral, athermal, and high-speed performance. IEEE J. Sel. Top Quantum Electron. 2013, 19, 127-137. [CrossRef]

15. Terada, Y.; Ito, H.; Nguyen, H.C.; Baba, T. Theoretical and experimental investigation of low-voltage and low-loss 25-Gbps Si photonic crystal slow light Mach-Zehnder modulators with interleaved p/n junction. Front. Phys. 2014, 2, 1-9. [CrossRef] 
16. Brosi, J.M.; Koos, C.; Andreani, L.C.; Waldow, M.; Leuthold, J.; Freude, W. High-speed low-voltage electro-optic modulator with a polymer-infiltrated silicon photonic crystal waveguide. Opt. Express 2008, 16, 4177-4191. [CrossRef] [PubMed]

17. O'Faolain, L.; Schulz, S.A.; Beggs, D.M.; White, T.P.; Spasenović, M.; Kuipers, L.; Morichetti, F.; Melloni, A.; Mazoyer, S.; Hugonin, J.P.; et al. Loss engineered slow light waveguides. Opt. Express 2010, 18, 27627-27638. [CrossRef] [PubMed]

18. Ponchak, G.E.; Papapolymerou, J.; Tentzeris, M.M. Excitation of coupled slotline mode in finite-ground CPW with unequal ground-plane widths. IEEE Trans. Microwave Theory Technol. 2005, 53, 713-717. [CrossRef]

19. Shin, J.H.; Sakamoto, S.R.; Dagli, N. Conductor loss of capacitively loaded slow wave electrodes for high-speed photonic devices. J. Lightwave Technol. 2011, 29, 48-52. [CrossRef]

20. Chen, H.W.; Kuo, Y.H.; Bowers, J.E. $25 \mathrm{~Gb} / \mathrm{s}$ hybrid silicon switch using a capacitively loaded traveling wave electrode. Opt. Express 2010, 18, 1070-1075. [CrossRef] [PubMed]

21. Li, G.L.; Mason, T.G.B.; Yu, P.K.L. Analysis of segmented traveling-wave optical modulators. J. Lightwave Technol. 2004, 22, 1789-1796. [CrossRef]

22. Jaeger, N.A.; Lee, Z.K. Slow-wave electrode for use in compound semiconductor electrooptic modulators. IEEE J. Quantum Electron. 1992, 28, 1778-1784. [CrossRef]

(C) 2016 by the authors; licensee MDPI, Basel, Switzerland. This article is an open access article distributed under the terms and conditions of the Creative Commons by Attribution (CC-BY) license (http://creativecommons.org/licenses/by/4.0/). 